\title{
Contingent Subject Trial Contact
}

National Cancer Institute

\section{Source}

National Cancer Institute. Contingent Subject Trial Contact. NCI Thesaurus. Code C142463.

Planned response to an anticipated but conditional event in a clinical trial. (CDISC) 